Article

\title{
Strategic Actions for Increasing the Submission of Digital Cadastral Data by the Surveying Industry Based on Lessons Learned from Victoria, Australia
}

\author{
Hamed Olfat ${ }^{1,2, *}$, Davood Shojaei ${ }^{1,2}$, Mark Briffa ${ }^{1}$, Susannah Maley ${ }^{1}$ and Abbas Rajabifard ${ }^{2}$ \\ 1 Land Use Victoria, Department of Environment, Land, Water and Planning, 2 Lonsdale Street, \\ Melbourne, VIC 3000, Australia; Davood.Shojaei@delwp.vic.gov.au (D.S.); \\ Mark.Briffa@delwp.vic.gov.au (M.B.); Susannah.Maley@delwp.vic.gov.au (S.M.) \\ 2 Centre for SDIs and Land Administration, Department of Infrastructure Engineering, \\ The University of Melbourne, Parkville, VIC 3010, Australia; Abbas.R@unimelb.edu.au \\ * Correspondence: Hamed.Olfat@delwp.vic.gov.au; Tel.: +61-3-9194-0513
}

Received: 12 December 2017; Accepted: 1 February 2018; Published: 4 February 2018

\begin{abstract}
Plan, as a digital cadastral data initiative, is a collaborative program between the land authorities and the surveying industry which aims to replace paper and PDF cadastral plans and surveys with digital data. ePlan is currently operational in Australia, New Zealand and Singapore. ePlan was introduced in the State of Victoria in 2011 and has been operational in this jurisdiction for 2D plans since 2013. On average, one ePlan application is currently submitted to a digital plan lodgment portal every two weeks. The low uptake of ePlan is caused by several technical and non-technical challenges. This paper provides an overview of cadastral information transitioning from paper to digital in Victoria. The research methodology to identify the challenges in Victoria for the adoption of ePlan is then described. This is followed by a discussion on the identified challenges. The paper then proposes a generic framework of strategic actions to increase the uptake of digital cadastral data based on the lessons learned from Victoria. The initiatives suggested by this framework to address the ePlan challenges in Victoria and increase its uptake are also introduced. The paper concludes with a direction for future research.
\end{abstract}

Keywords: digital cadastral data; ePlan; strategic actions; uptake; 3D cadastre; Victoria; Australia

\section{Introduction}

Cadastre, which includes land's geographical and legal information (rights, restrictions and responsibilities), is the base and core component of any Land Administration System (LAS). The cadastre is also one of the fundamental datasets in an integrated, multi-level hierarchy of interconnected Spatial Data Infrastructures (SDIs) based on partnerships at corporate, local, state/provincial, national, multi-national (regional) and global levels [1].

Within the SDI context, the cadastral data needs to be provided in digital format so that they can be shareable, discoverable, retrievable and downloadable. The limitations of the traditional approach (paper/PDF-based plans) of a cadastral survey such as plan lodgments and registration have caused several issues to the Australian land development process [2]. This is why a paperless and fully automated cadastral infrastructure has been highly recommended by the Cadastre 2034 national strategy developed by the Intergovernmental Committee on Surveying and Mapping (ICSM) [3], which is responsible for providing leadership, coordination and standards for surveying, mapping and national datasets in Australia and New Zealand.

To this end, an ePlan Working Group (eWG) was formed by ICSM, which developed a national data model to transfer digital cadastral data between the Australian surveying industry and land 
registries in 2009 [4]. In 2011, an ePlan Protocol was developed to map the components of the ePlan data model to LandXML data format [5]. The ePlan LandXML file is an intelligent digital file which contains cadastral plan and survey information that includes survey measurements, dimensions of the parcels, interests in land (e.g., easements and restrictions), land parcel descriptions, administrative information (e.g., locality), owners' corporation schedules, survey marks, traverses, radiations, connections to title, annotations, plan approval status and stakeholders' signatures.

2D ePlan is currently operational in New South Wales (NSW) and Victoria. Other jurisdictions have also participated in the eWG and are investigating their options. New Zealand is also part of the eWG. The New Zealand e-Cadastre survey process was streamlined through the Landonline system, which was rolled out in the 2000s. This was achieved by: (1) surveyor extracts digital data (including coordinates) and images of old plans for area of survey; (2) digital data imported into surveyor's software; (3) survey undertaken (calculations and field work); (4) data for new survey exported; (5) surveyor imports data into Landonline, adds any missing elements, generates plan image, and pre-validates (internal consistency and comparison against existing data); (6) surveyor formally submits survey; and (7) Land Information New Zealand (LINZ) staff validate and approve survey, data becomes official and new coordinates generated [6,7]. The cadastral data is transferred via the ePlan LandXML format. New Zealand mandated ePlan in 2007 under section 42 of Cadastral Survey (Compulsory Lodgment of Digital Cadastral Survey Datasets) Order 2007 [8].

The Singapore Land Authority (SLA) also joined the eWG in 2013, and it has adopted the ePlan LandXML Protocol for its cadastral survey modelling and electronic lodgments [9,10]. Singapore (SG) LandXML Mapping and Structure technical paper was officially released in 2017 [11]. The SLA new cadastral system, namely Cadastral Survey Management System (CSMS), which is currently under development, has two main components including Registered Surveyors' (RS) Portal and SLA Inspection and Approval [12]. Registered surveyors will prepare their SG LandXML submissions in office. The RS Portal will provide facility for registered surveyors and their assistants to pre-validate their submissions, and to generate cadastral plans from the submitted SG LandXML to receive updates and consultations from SLA. After passing the RS Portal, the job submission will be passed on to inspection officers at SLA based on certain processing workflow for detailed inspections and cadastral validations. As all submissions will be validated upfront at the Portal, no major errors should be expected when reaching the SLA. The Chief Surveyor will approve the plan when the jobs are ensured to be in proper order. The cadastral data from the SG LandXML submissions will then be stored in the database, which is designed based on the SG Land Administration Domain Model (LADM) [13]. The LADM passed the final vote towards becoming an international standard on the November 1, 2012 [14]. Similar to Singapore, there are other countries that have moved towards implementing the LADM model for their digital cadastre. Examples of such implementations can be seen in Cyprus, Canada, Croatia, Brazil, South Africa, and Portugal [15-20]. Most of these implementations, however, are limited to research and development in academia at this stage [14].

Apart from the countries developing ePlan LandXML or LADM, the digital cadastre concept has been well developed in other countries. According to literature, the examples include Denmark and Spain. In Denmark, the licensed land surveyors use a portal to prepare applications for cadastral changes in a way that the data can be used directly for updating the cadastral databases at the Cadastral Authority at the National Survey and Cadastre. The system is called MIA. Data is transferred directly into a standardized format (XML) from the MIA system to the cadastral data handling system at the National Survey and Cadastre. This system is called miniMAKS and presents the data prepared by the licensed land surveyors and enables the employees of the National Survey and Cadastre to verify that the relevant approvals are obtained before accepting the proposed changes to the cadastre [21]. Spanish cadastre uses File for Interchange of Cadastral Cartography (FICC), an ASCII format, which reflects the geometry and features of the entities of cadastral elements. Cadastral Virtual Offices have been created which provide services to make cadastral administration more efficient and effective. They have given society free, secure, easy, rapid, 24-hour access to the cadastral database, which is updated daily [22]. 
ePlan was introduced in the State of Victoria in 2011 and the state's digital lodgment portal, SPEAR, which is explained in Section 2, incorporated ePlan functionality in 2013. Following the progress of the Victorian ePlan implementation, the research problem and questions underpinning this paper have been defined as follows.

\subsection{Research Problem}

Although the Victorian ePlan services aim to facilitate the ePlan creation and submission process for surveyors, on average only one ePlan application is currently submitted to SPEAR every two weeks. Accordingly, the uptake of ePlan being presently low in Victoria has been defined as the research problem to be investigated in this paper.

\subsection{Research Questions}

In considering the research problem, two key research questions emerged, as follows:

1. What are the main challenges impacting the low uptake of ePlan?

2. What strategic actions can be defined to overcome the challenges causing the low uptake of ePlan?

The remainder of this paper is structured as follows. An overview of cadastral information in Victoria and its transition from paper to digital is first provided. Then, a research methodology for identifying the ePlan challenges is described in Section 3. This is followed by an overview of the ePlan implementation challenges in Section 4 (responding to research question 1). The findings are then used in Section 5 to develop a framework of strategic actions to address the ePlan challenges and increase the uptake of ePlan (responding to research question 2), which could conceivably be used to support the adoption of the submission of digital cadastral data within other Australian jurisdictions and countries. Finally, Section 6 concludes the paper with a direction for future research.

\section{Victorian Cadastral Information Transitioning from Paper to Digital}

Figure 1 illustrates the Victorian cadastral information transitioning from paper to digital over time. Before the 1990s, cadastral plans were all lodged in paper. Vicmap Property, the State Digital Cadastral Database (DCDB), was created in the early 1990s from the digitization of paper-based map records held by Melbourne Water (metropolitan area) and the State government (rural area). Vicmap Property comprises more than 3 million land parcels and associated property attributes, such as lot and plan number, and crown description, in the State of Victoria.

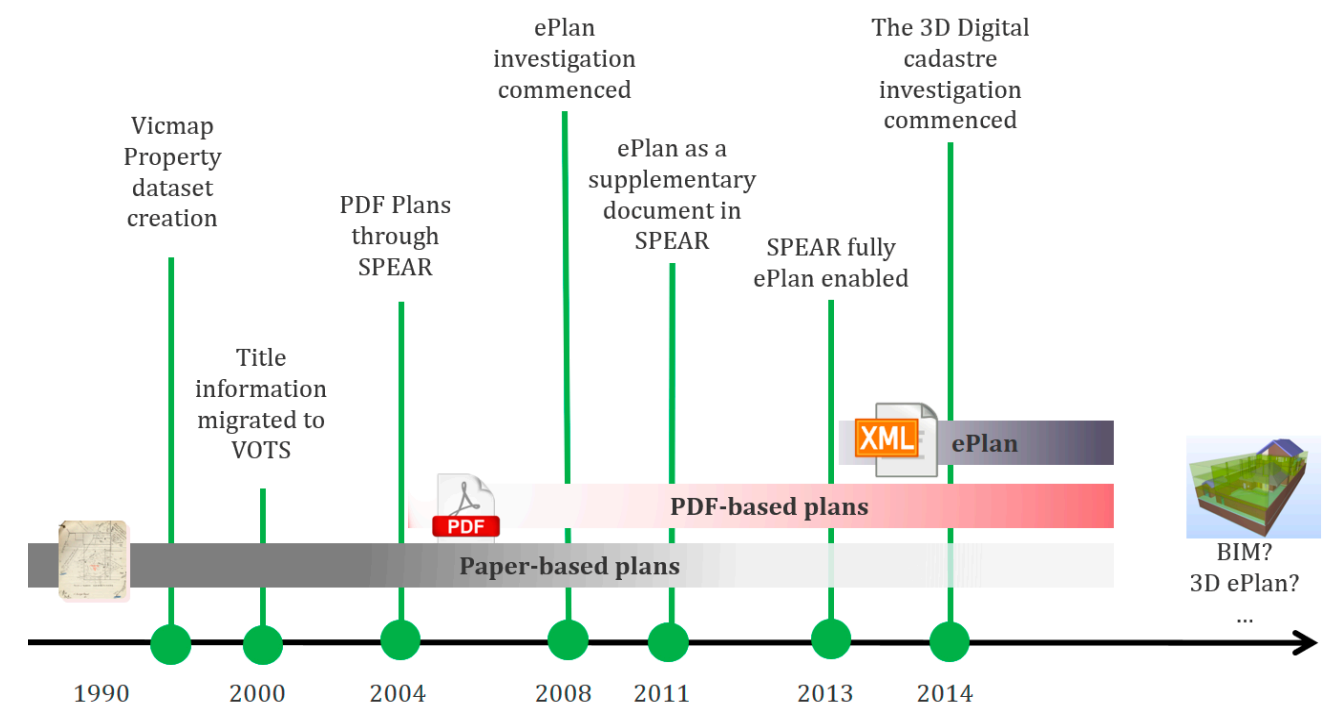

Figure 1. Victorian cadastral information (paper $\rightarrow$ digital). 
Land title information was migrated from paper to the Victorian Online Title System (VOTS) in 2000. VOTS contains a record of all Victorian titles registered under the Torrens System [23]. The system is maintained by Land Use Victoria (LUV) and is used to accept, create and register land transaction lodgments, and to update land holdings and registered interests on title as well as create new titles.

Prior to the launch of the Surveying and Planning through Electronic Applications and Referrals (SPEAR) pilot in 2004, subdivision applications could only be processed via paper. Diagram (a) in Figure 2 illustrates this process. This was generally a lengthy and protracted process that was instigated by the surveyor, on behalf of their client (developer). The process and application milestones are well defined by the Planning and Environment Act 1987 [24] and Subdivision Act 1988 [25], however achieving these milestones, in a paper environment, where there are multiple stakeholders involved in the decision-making process led to delays, errors, and poor transparency between the interested parties.

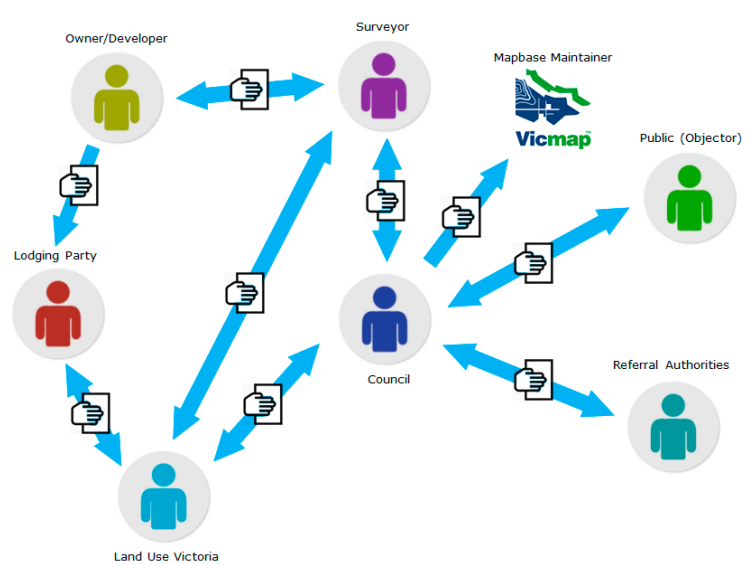

(a) Subdivision process before SPEAR

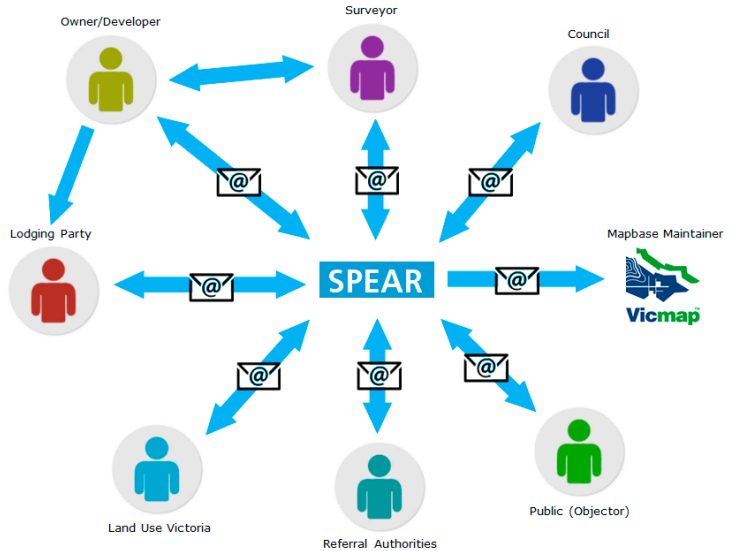

(b) Subdivision process after SPEAR

Figure 2. Subdivision process before SPEAR (Surveying and Planning through Electronic Applications and Referrals) (diagram a) vs. after SPEAR (diagram b).

Although there was a lot of scope to improve efficiencies throughout the life of the application, there was no means of implementing these changes for the benefit of all parties, due to the technology constraints of a pre 'world wide web' era.

SPEAR revolutionized the way subdivision applications were handled, by introducing online end-to-end processing and tracking of plan applications from their initial submission with local government, right through to registration at LUV. Diagram (b) in Figure 2 illustrates the subdivision process after the introduction of SPEAR. A surveyor can use SPEAR to apply for any plan-based dealing under the Subdivision Act 1988 [25], and the planning permit to subdivide under the Planning and Environment Act 1987 [24].

SPEAR introduced invaluable transparency and accountability to the subdivision application process by streamlining the approval process for plans of subdivision, and the associated planning permit to subdivide. The system is now being used by all 79 Victorian local governments, 200 surveying firms, 74 referral authorities, and LUV, which, in total, represents over 4000 users to view the progress of applications.

According to Figure 1, the investigations to the ePlan project commenced in 2008 in Victoria. LUV collaborated with the ICSM eWG on developing a national data model to cover all Australian jurisdictions' cadastral and survey requirements. In 2011, SPEAR enabled surveyors interested in ePlan to upload an ePlan LandXML file along with their PDF application. From 2011 to 2013, ePlan was piloted in Victoria by LUV, the surveying industry and software vendors. In May 2013, SPEAR incorporated ePlan services including visualization, validation, data viewer and data download, which are briefly discussed below [26]: 
- ePlan Visualization Service-This service converts the ePlan LandXML file into PDF. The PDF plan forms part of the legal title in Victoria. The automatic visualization of an ePlan file is fundamental in streamlining the processes and dissemination of digital cadastral data.

- ePlan Validation Service-This service identifies errors and potential problems in plans at an early stage of preparation, and allows surveyors to correct these issues prior to the LUV examination process. This will result in a reduction in the number of refusals and requisitions received by surveyors sent by LUV. SPEAR currently has 130 ePlan validation rules which cover four main areas, the 'ePlan schema compliancy', 'survey accuracy (e.g., parcel area, parcel observations closure)', 'survey examination rules (e.g., appropriate title connections)' and 'metadata completeness (e.g., easement purpose)'.

- ePlan Data Viewer-This service provides a tabular view of both the graphical and textual data that can be used to review the content of an ePlan. This assists the surveyors to check the correctness of the ePlan data that has been uploaded in the LandXML file.

- ePlan Data Download Service-This service is available through the LASSI-SPEAR application. LASSI-SPEAR is a free web mapping application that allows users to search and download survey information. The digital data download service allows users to draw a polygon on the Mapbase (i.e., Vicmap Property) and download a digital file in ePlan LandXML format that contains parcel line works, administrative areas, datum, location addresses, road abuttals, survey marks and monuments. The downloaded ePlan file can be imported into surveying software packages to pre-populate the data for ePlan preparation, a saving for the surveyor.

Currently, three software vendors including LISTECH, Civil Survey Solutions and Geocomp Consulting support the Victorian ePlan in their respective software packages namely LISCAD, Stringer ePlan and ePSALON.

All 2D plans under the Subdivision Act 1988 [25] are supported in ePlan. However, strata plans (building subdivision plans) which include overlapped ownership rights are not yet supported. As indicated in Figure 1, the investigations around the 3D digital cadastre to support the building subdivisions in ePlan format commenced in 2014.

Following the research problem discussed in Section 1.1, the uptake of ePlan is presently low in Victoria. To identify the main challenges impacting the low uptake of ePlan, a methodology has been implemented which is discussed in the next section.

\section{Methodology}

According to Myers [27], qualitative research methods were developed in the social sciences to enable researchers to study social and cultural phenomena. The strengths of qualitative research methods lie in their usefulness for understanding the meaning and context of the phenomena studied, and the particular events and processes that make up these phenomena over time, in real-life, natural settings [28]. Mack et al. [29] also summarized two main advantages of qualitative research methods. The first advantage is using open-ended questions, which give participants the opportunity to respond in their own words. Open-ended questions have the ability to evoke responses that are meaningful and culturally salient to the participant, unanticipated by the researcher, and rich and explanatory in nature. Another advantage of qualitative research methods is that they allow the researcher the flexibility to probe initial participant responses-that is, to ask 'why' or 'how'.

Following the above-mentioned advantages of qualitative research, this type of research methodology was chosen for identifying the ePlan implementation challenges. Among the available qualitative research methodologies such as interview, questionnaire, focus groups, etc. [30], a face to face interview with ePlan users (surveyors) was selected, because this methodology allowed:

- no significant time delay between question and answer so the interviewer and interviewee can directly react on what the other says or does [31],

- closer interaction with surveyors for presenting and discussing ePlan, 
- obtaining detailed information about personal feelings, perceptions and opinions on ePlan adoption,

- receiving subsequent and immediate feedback on ePlan process and services, and

- engaging a wide range of surveying firm staff members, such as licensed surveyor, quality assurance manager and draftsman that could be impacted by ePlan services, in the discussions.

An expression of interest was distributed among more than 200 surveying firms in Victoria, and 26 showed interest to be part of the interview process. The ePlan team approached each surveying firm individually to ensure that surveyors were comfortable to share their views and concerns on ePlan challenges. Table 1 shows the details of interviews participants. It also indicates the size of each surveying firm. The firms were categorized by the number of SPEAR users at each organization (including multiple offices, if applicable), as following:

- $\quad$ Small-<10

- Medium-10 to 20

- $\quad$ Large-21 or more

Table 1. Participants of face to face interviews.

\begin{tabular}{|c|c|c|c|c|c|c|c|}
\hline & Surveying Firm & Firm's Size & $\begin{array}{l}\text { Number of } \\
\text { Participants }\end{array}$ & & Surveying Firm & $\begin{array}{l}\text { Firm's } \\
\text { Size }\end{array}$ & $\begin{array}{l}\text { Number of } \\
\text { Participants }\end{array}$ \\
\hline 1 & Bosco Jonson & Large & 2 & 14 & KLM Spatial & Medium & 4 \\
\hline 2 & TGM Group & Large & 1 & 15 & Thomas \& George & Small & 2 \\
\hline 4 & Barker Monahan & Small & 3 & 17 & B R Smith Surveyors & Small & 1 \\
\hline 5 & Spiire & Large & 2 & 18 & $\begin{array}{c}\text { Alexander Symonds Surveying } \\
\text { Consultants }\end{array}$ & Small & 3 \\
\hline 8 & Charter Keck Cramer & Small & 4 & 21 & Tomkinson Group & large & 3 \\
\hline 9 & Beveridge Williams & Large & 3 & 22 & Meridian Land Surveyors & Small & 1 \\
\hline 10 & SMC Land Surveyors & Small & 3 & 23 & EDM Group & Medium & 3 \\
\hline 11 & Moonland Group & Small & 1 & 24 & Calibre Consulting & Small & 5 \\
\hline 12 & Cardno & Small & 2 & 25 & JH Surveying & Small & 1 \\
\hline 13 & Smith Land Surveyors & Medium & 3 & 26 & Hellier McFarland & Medium & 4 \\
\hline
\end{tabular}

Prior to the interviews, the ePlan team created an ePlan for each interview session using the simple cadastral plans produced by the participating surveying firms. Using this ePlan file, the ePlan visualization, validation, and data viewer services within SPEAR were demonstrated. The interviews began with an introduction to the ePlan implementation progress and online services. The following open-ended questions were then asked during the interviews:

1. What is your end-to-end cadastral subdivisional process?

2. How many cadastral plans are prepared by your firm annually? How complex are they (e.g., 2-3 lot plans, greenfield subdivisions, high rises, etc.)?

3. What software packages do you use for cadastral plans computation and drafting?

4. How long do you normally spend on drafting a cadastral plan and its filed notes?

5. What impacts do you think will be applied to your subdivisional process by adopting ePlan?

6. What do you think about the plan visualized by SPEAR as compared to your originally drafted plan?

7. How do you think the ePlan should benefit your organization?

8. What barriers do you currently see in adopting ePlan?

The outcomes of the interviews were analyzed and the identified ePlan challenges were categorized into five main groups which are discussed in the next section. 


\section{Identified Challenges of ePlan Implementation in Victoria}

\subsection{Challenge 1: Surveyors' Adopting a New Business Process}

The interviews showed that ePlan is basically seen by surveyors as an enormous change to their subdivisional practices, which is introducing extra cost to the surveyors' business. The cost includes two parts: (1) software/training cost; and (2) business process change cost.

Surveyors need to purchase ePlan software. This results in enforcing extra cost to the surveyors' business. The surveyors must also undertake training to acquire the skills required to create ePlans. Although most of training sessions are free, the surveyors need to spend time out of their office for training purposes.

The participants believed that ePlan is going to change their subdivisional process dramatically since they need to follow the process of creating the ePlan and then submitting it to SPEAR, instead of preparing the PDF plan. They were reluctant to change their existing and well-established processes.

\subsection{Challenge 2: Limitations of the ePlan Visualization Service}

The quality of the current visualization service was criticized by the interviewees as it cannot efficiently draft complex plans, including appropriate support to creating enlargement diagrams, a key component of plan presentation. Feedback received from the participants also indicated that in general, surveyors would like to control the plan presentation of the PDF visualized from an ePlan.

The limitations of the ePlan visualization service have resulted in a negative impression from surveyors. The surveyors expect high quality visualized PDFs. It must meet their surveying standard as the plan is a representation of their work, and that of their surveying firm. Their clients have an expectation of the quality of work being produced from the hired surveyor.

\subsection{Challenge 3: Commercial Benefits of ePlan Being Intangible to Land Surveyors}

Majority of participants believed that ePlan mainly benefits the land authority and not the surveyors. Unlike SPEAR, ePlan benefits are not clearly obvious for land surveyors. There is currently no commercial incentive considered for surveyors to adopt ePlan in Victoria. Whereas in NSW, incentives have been introduced by Land and Property Information (LPI) for surveyors to take up ePlan. These incentives are:

- Quicker examination-it takes a traditional plan approximately 12 working days to be allocated to a plan examiner, however, for ePlans this is reduced to an average of five working days. Although plan lodgments still cost the same, the savings of holding costs for developers are significantly reduced.

- LPI provides ePlan files (if available) to surveyors for plans that are used to start a new survey. This eliminates the need for surveyors to enter the geometry and survey mark data into their survey software when starting a new survey. This reduces time and eliminates data entry error.

- LPI introduced a requisition fee for any issue in cadastral plans before registration as of 1 January 2017, but if the requisition relates to an ePlan and not related to survey or regulations, the fee is waived.

According to these incentives in NSW, the ePlan uptake is higher in this jurisdiction compared to Victoria.

\subsection{Challenge 4: Building Subdivisions in ePlan Not Fully Supported}

2D plans under the Subdivision Act 1988 [25] are currently supported in ePlan. This includes boundaries defined by buildings and building returns on 2D plans, however building subdivision plans with overlapping ownership rights are not supported. According to the interview outcomes, this has resulted in the reluctance of surveyors adopting ePlan at this stage. They prefer to implement ePlan when the whole process of subdivision is supported. 


\subsection{Challenge 5: Abstract of Field Records in ePlan Not Fully Supported}

Since ePlans must be connected to the Map Grid of Australia (MGA) 1994 datum, the surveyors are highly recommended to include a subset of Abstract of Field Records (AFR) in their ePlan, such as survey control marks, traverses and radiations. The Victorian AFR normally includes significant details relating to control marks, reference marks, traverse and radiation observations, title boundaries and occupation e.g., fences and walls. To illustrate these details, surveyors undertake distortion and exaggeration in AFR diagrams. These complexities cause issues for the ePlan visualization service to create a satisfactory AFR diagram. The AFR is mainly used by plan examiners and surveyors to re-establish the survey. Accordingly, surveyors are required to submit their AFR to SPEAR in PDF format. Similar to challenge 4, this can result in demotivating surveyors to adopt ePlan as they prefer to implement ePlan when the process of the subdivision is fully supported.

The next section introduces a framework of strategic actions to address the identified ePlan challenges and increase its uptake.

\section{A Framework of Strategic Actions for Increasing the Submission of Digital Cadastral Data}

Following the analysis of the interview findings, a framework comprising four strategic actions has been developed to address the challenges raised by the surveyors in the adoption of the submission of digital cadastral data. This framework, as shown in Figure 3, is generic and could be applied to other land authorities in the world dealing with digital cadastral data adoption.

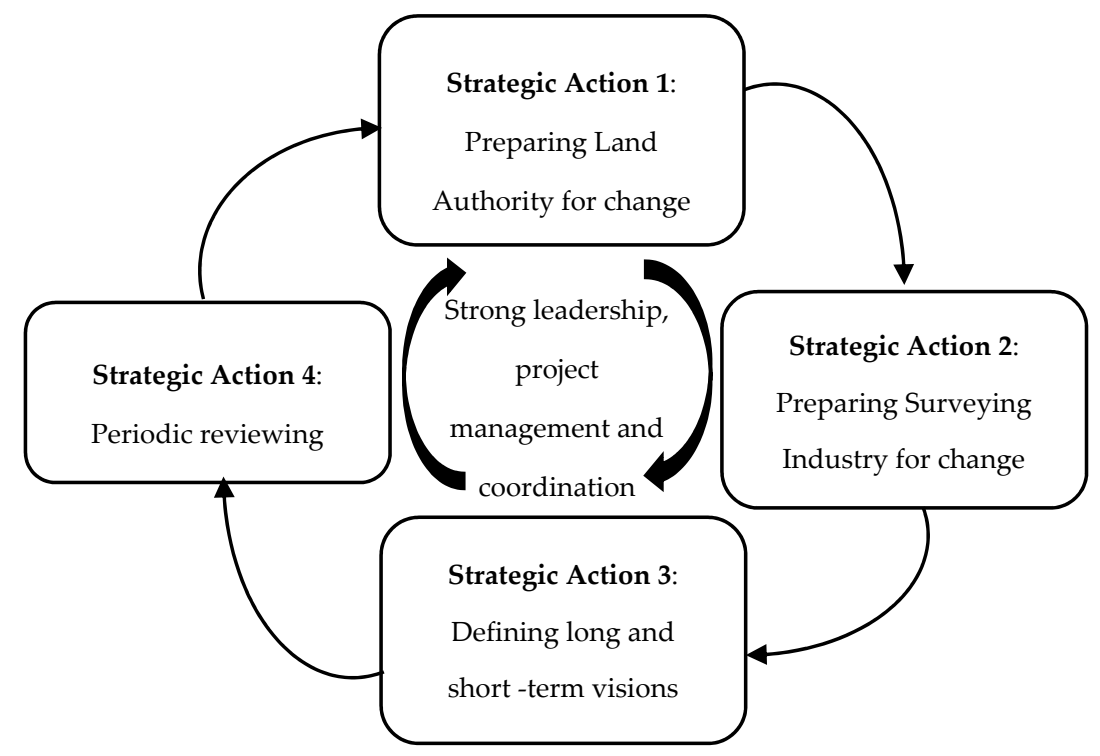

Figure 3. A framework of strategic actions to increase the adoption of the submission of digital cadastral data.

The four strategic actions, relevant initiatives and the aspects of leadership, project management and coordination are discussed below.

\subsection{Preparing Land Authority for Change}

Land authority needs to prepare for change by fully understanding the requirements to digital cadastral data adoption for the present, as well as considering the future in relation to the collection of digital cadastral data, and the impacts to existing infrastructure, policies, and processes. This strategic action has been developed to address the challenges 2, 3 and 5 discussed in Section 4 . The following initiatives are being undertaken to prepare LUV for the change. 
5.1.1. Extending the SPEAR Service Desk Role to Support Surveyors with ePlan Preparation Issues

The SPEAR Service Desk team has extended its role to support ePlan. The SPEAR Service Desk team are fully resourced with skills and knowledge required for plan and survey examination processes. The ePlan applications lodged at LUV are immediately picked up from the lodgment queue by the SPEAR Service Desk and are initially examined to identify any technical ePlan issues, which can then be raised with the surveyor or forwarded to the ePlan team for resolution.

\subsubsection{Undertaking of a Pilot Project to Upgrade the Spatial Accuracy of the Digital Cadastral Mapbase}

The current accuracy of Digital Cadastral Mapbase is low in some areas of Victoria [32]. Due to this, the Office of Surveyor General Victoria, in collaboration with the ePlan team have coordinated a pilot project to determine the best approaches to improving the spatial accuracy of Victoria's Digital Cadastral Mapbase using ePlan data. The pilot project is investigating (1) the back-capture of survey information from paper plans and CAD drawing files converted into ePlan; and (2) a suitable methodology for the establishment and maintenance of Mapbase via least squares adjustment of the back captured information.

The outcomes of this project will highlight the importance of the surveying industry's contribution to updating the spatial accuracy of the Mapbase, by submitting ePlan files instead of PDF files through SPEAR to LUV. Surveyors will be encouraged to submit ePlans within spatially upgraded areas and to download data from the Mapbase for their subsequent surveys.

\subsubsection{Developing an ePlan Visualization Enhancement Tool}

A new web-based tool has been recently developed for surveyors that has functions to improve the presentation of the PDF visualized from the ePlan data. This tool, named the ePlan Visualization Enhancement Tool (VET) provides surveyors with functionality to:

- place labels and arrows

- define new sheets

- create enlargement diagrams

- exaggerate lines

- $\quad$ support subsequent changes made to an ePlan file.

This tool has been demonstrated to surveyors in several conferences and workshops. Surveyors can now use this tool to enhance the prepared plan produced by the visualization service. In the current practice (paper and PDF plans), surveyors spend several hours to days to draft their plans, depending on the complexity of the plan. Time will be saved using a combination of the ePlan visualization service and VET.

\subsubsection{Extending Systems to Derive Benefit from 2D/3D Digital Cadastral Data}

Figure 4 shows a complete future workflow that would benefit from the submission of ePlans. The core component of this workflow is the spatially-accurate Digital Cadastral Mapbase, which will be developed to enable surveyors to download cadastral data in ePlan format using a web/mobile application.

The ePlan web/mobile application will be developed to enable surveyors to access, search, download and query registered ePlans stored in an Oracle database. This database is currently under implementation. Apart from the basic functions (e.g., pan, zoom, identify, etc.), this application aims to assist surveyors in constructing their surveys by re-using existing ePlan data through the following main functions:

- Displaying ePlans on the Digital Cadastral Mapbase

- Retrieving registered ePlans from the database and displaying the data in different layers (e.g., lot, easement, traverse, radiation, survey mark, etc.) 
- Using query builder to search ePlans based on user-defined queries

- Downloading ePlans in a specific administrative area (e.g., Local Government Area) or user-defined area (freehand polygon)

- Navigating from the user's position to survey control marks, reference marks and boundary points.

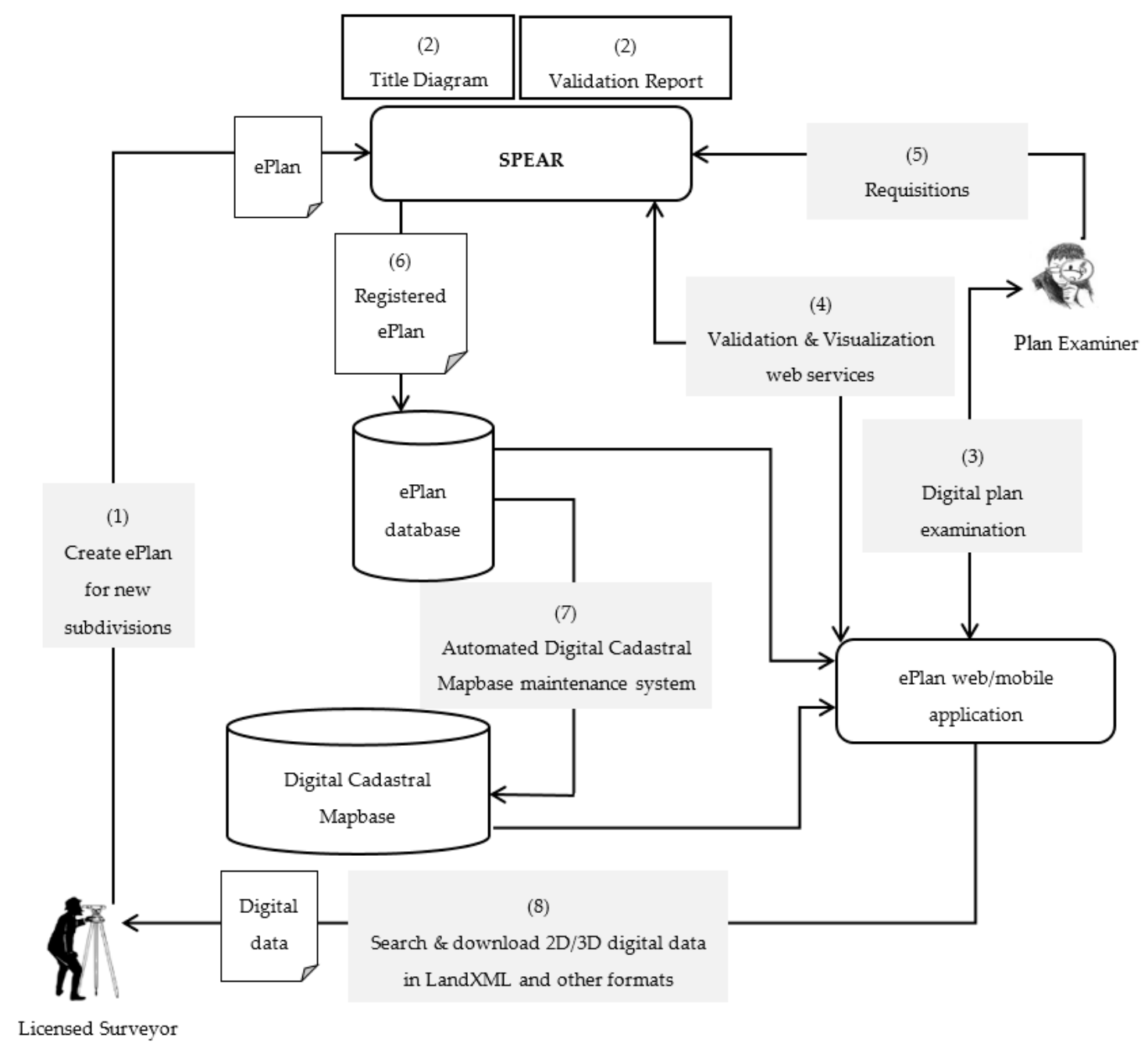

Figure 4. Future ePlan workflow.

This web/mobile application will deliver the tangible benefits of ePlan to surveyors in both their office and in the field. It will be used on-site on mobile devices to simplify the process of locating survey details (e.g., a survey mark) in registered surveys available in ePlan format. Through the data download service, the application will have functionality to import the existing accurate plan and survey data directly into a computations package and have it validated on-site. This replaces the existing processes of using paper/PDF abstract of field records.

Automation of validating the survey re-establishment will also be developed within the ePlan web application to facilitate the survey examination process. The examination checks that are not part of the ePlan validation service will be programmed into this application, resulting in efficiency gains in ePlan applications from lodgment to registration.

According to Figure 4, the new cadastral plan will be submitted to SPEAR in ePlan format (1). Like current practice, SPEAR provides the surveyor with a validation report and a visualized title diagram (2). These ePlan services have been explained in Section 2. Plan examiners will digitally 
examine the lodged plan through an ePlan web application discussed above (3). Through validation and visualization web services, the plan examiners will be able to validate and visualize the cadastral plans as part of their examination process (4). The plan examiners will be able to send the requisitions to surveyors through SPEAR (5). Once the ePlan is registered, a copy of the LandXML file will be sent to the ePlan database (6), and an automatic process will be required for the Digital Cadastral Mapbase adjustment and update (7). The outcomes of the back-capture project will be utilized in building such a process. To re-establish the subsequent surveys, the surveyors will be able to search the existing registered ePlans using the ePlan web/mobile application and download 2D/3D digital data in LandXML and other formats to be used in the field and office (8). The ePlan application will be connected to the ePlan database and the Digital Cadastral Mapbase to show both the parcel corners adjusted by LUV and the original survey information supplied by the surveyors.

Among the components shown in Figure 4, the ePlan database has been developed using Oracle technology and is currently under test. The development of an ePlan web/mobile application has commenced recently. A team has also been formed by LUV to modernize the Digital Cadastral Mapbase.

\subsection{Preparing Surveying Industry for Change}

In parallel to the changes in land authorities, the surveying industry needs to prepare for supporting the change in terms of submitting cadastral data. This strategic action has been developed to address the first challenge discussed in Section 4. Some of the important LUV initiatives in this regard are discussed below.

\subsubsection{Industry Engagement}

In 2015, an ePlan engagement program with the surveying industry commenced to investigate the subdivisional processes of surveying firms. This approach enables the ePlan team to gain better understanding of how ePlan could potentially fit into the existing surveying processes. The program also enables surveyors to familiarize themselves with ePlan and to provide feedback to the current ePlan services, and to participate in future developments of ePlan. Two rounds of this program have been undertaken so far and it will continue in the future.

\subsubsection{Piloting ePlan}

LUV is planning to pilot VET and the associated ePlan services with interested surveying firms in $2018 / 2019$. The purpose of the pilot is to enable the pilot participants to test the functionality of the ePlan services, in conjunction with their surveying software that supports the creation of ePlan. All participants will require formal training and ongoing technical support throughout the pilot. The success of the pilot will be determined by the severity of the defects and any requested enhancements from the pilot participants. Any defects raised in the pilot will be addressed by the ePlan team. Defects may also be raised with the ePlan packages offered by software vendors, which could also impact the success of ePlan. These defects would require development from the software vendors.

The pilot will be offered to 20 surveying firms, with the expectation they individually submit 10 2D ePlans containing no more than 10 lots. This approach is to confirm the ePlan visualization service, VET and that the surveying software can efficiently handle small scaled plans. It will also allow the pilot participants to develop the new skills and knowledge required to produce basic ePlans before embarking on the more complex and detailed plans in the future. During the pilot, the ePlan team will be making further enhancements to the ePlan services to better facilitate the creation of ePlans for the larger and more detailed plans. As an incentive to surveying firms to join the pilot, 2D ePlans will be examined at lodgment and processed to registration. The normal waiting times before plans are examined is approximately 15 business days. 


\subsubsection{Undertaking ePlan Case Studies}

Defining a case study with data from large surveying firms has been another initiative to demonstrate the benefits of ePlan to the industry and to prepare them for change. A case study was undertaken with data provided by SMEC Australia, one of the largest surveying firms in Victoria. The aim of SMEC's case study was to align ePlan with their current business practices and product quality. The outcomes of this case study, and the potential benefits of ePlan to a surveying firm have been presented to the surveying industry. These case studies will continue in the future to identify the possible issues in implementing ePlan within surveying firms.

\subsubsection{Conducting ePlan Training Workshops}

The ePlan team and the software vendors conduct several workshops for surveying firms to familiarize them with the ePlan software packages. The entire process of creating ePlan is demonstrated in these workshops, providing the knowledge and skills to enable surveyors to create their first ePlan, and to validate and visualize it.

\subsection{Defining Long and Short-Term Visions}

Land authorities need to have a detailed road map in the adoption of digital cadastral data. This should define both long and short-term vision statements and relevant milestones, projects and timeframes. The roadmap informs surveying firms the commitment of planned changes to enable them to prepare in the adoption of the submission of digital cadastral data.

Accordingly, LUV's digital cadastral vision statements have been defined as following:

- $\quad$ Long-term vision: Implement ePlan for all Victorian cadastral plans and surveys by 2025.

- Short-term vision: Provide the infrastructure and services to enable the submission and registration of ePlan for all 2D Victorian cadastral plans by 2020.

A key component of the long-term vision is supporting the strata plans in digital format, to address challenge 4 in Section 4. This is following the Cadastre 2034 national strategy developed by ICSM in 2014. This strategy has a vision to develop a cadastral system that enables people to readily and confidently identify the location and extent of all rights, restrictions and responsibilities (RRRs) related to land and real property [3].

As part of its long-term vision, in 2014 LUV commenced resourcing the research into designing and implementing a 3D digital cadastre, focusing on providing more efficient and effective services to the land and property industry. Over the past three years, among the technical, legal and institutional aspects of a 3D digital cadastre [4,33], the focus has mainly been on investigating the technical aspects including 3D data visualization, validation and modelling to support building subdivision plans in ePlan [33-35]. The legal and institutional aspects have not yet been researched. The current Act, the Subdivision ACT 1988 [25], allows registration of overlapped RRRs (building subdivisions) in Victoria and they are registered in an analogue (paper/PDF-based) approach. However, the registered data cannot be used for creating 3D models of RRRs. A review of legislation is required that encourages architects and surveyors to provide their 3D legal models when they submit building subdivision plans. Understanding the institutional issues in the context of a 3D digital cadastre also necessitates the development of strategies to support the change in processes [36]. The institutional aspect looks at what changes are required to existing processes and roles to facilitate implementation.

The institutional, technical and legal aspects of a 3D digital cadastre should link together to clarify the connection and relationship of different areas of responsibility to build a comprehensive framework for implementation. Again, there is a need for identifying the major milestones to achieve the long-term vision. This can be in line with the milestones suggested by Kalantari and Rajabifard [37] including (1) defining the scope of the 3D cadastre to include only high-rise and complex developments; (2) identifying the champion to lead the project; and (3) considering the technical requirements and capacity building in accordance with needs of stakeholders. 
The short-term vision is mainly concerned with supporting 2D plan based applications in digital format. As part of this vision, LUV has decided to follow the New Zealand strategy to support a hybrid combination of intelligent digital data for the survey header (LandXML) and PDF for the plots of buildings. This will be an initial approach for supporting strata plans in ePlan until the long-term vision is realized.

\subsection{Periodic Reviewing}

Any periodic change, particularly in the early stages, is likely to be highly dynamic with people, processes and organizations, all in varying stages of transition [38]. As a result, the last strategic action, periodic reviewing, is to ensure that strategic decisions and operational and technical actions remain necessary and relevant.

As time goes on, the technologies related to 2D and 3D digital cadastral data will change and evolve. Legislative and Registrar's requirements may also change over time. Periodic reviews will better support each subsequent implementation phase by identifying new technology and legislative changes, areas of success and continued challenges before moving to the next phase.

\subsection{Leadership, Project Management and Coordination}

As illustrated in the core of the framework in Figure 3, strong leadership, management and the coordination of tasks is required, particularly in a transformational project such as the implementation of ePlan. Leadership is critical in terms of defining a clear vision that impacts the behavior of the surveying and property industry and the processes of land authorities. The vision must be well defined and communicated with all key stakeholders. Vision statements and a roadmap will provide those affected with a clear picture as to future. A land authority, such as LUV must provide clear leadership and direction to the surveying and property industry in the implementation of ePlan. Communicating the key drivers and benefits to ePlan is critical in formulating a message to industry.

The management and coordination of implementing ePlan requires careful strategic planning. It must be focused on delivering on the vision statements and the tasks related to meeting the milestones within the roadmap. This will require well-managed resources that works collaboratively within its own land authority, which includes Office of Surveyor General, IT, and with plan and survey examiners. It must also engage with the surveying industry.

\section{Conclusions and Future Directions}

This paper reviewed the current status of the digital cadastral data (ePlan) initiative in Victoria. A face to face interview methodology confirmed that surveyors are reluctant to change their well-established processes and adopt ePlan because of a number of technical and non-technical challenges discussed in this paper.

The surveyors that have adopted ePlan acknowledged some of the ePlan advantages, such as pre-lodgment checks, using the validation service, and eliminating the drafting step from the plan preparation process by using the visualization service. However, they are not able to re-use the digital data created by other surveyors. Re-using ePlan data would significantly facilitate the creation of subsequent surveys as the required information, such as survey marks, traverse and radiation observations, title boundaries, easements, restrictions and administrative details can all be pre-populated in software packages from an existing ePlan. In the current practice, surveyors must use the PDF/paper abstract of field records and re-draw the plan and survey data to re-establish the subsequent surveys.

From LUV's perspective, ePlan would be similar to any other recent technological change, which may have impacted the current surveyors' business (e.g., building information modelling, point cloud, unmanned aerial vehicles, etc.). However, it was understood from the findings that surveyors would resist adopting ePlan until they see gains in time/cost saving or financial benefits (e.g., improved plan registration timeframes), or ePlan is mandated by the government. 
The findings led to the development of a framework of strategic actions to address the identified challenges to increase the uptake of ePlan. Each strategic action proposed initiatives, with some already in development. While it is difficult to generalize from the findings of a jurisdiction alone, the findings could potentially contribute to introducing digital cadastral data adoption by other Australian jurisdictions or other countries. Apart from the publications and presentations out of the Victorian ePlan project, the LUV ePlan team will share the findings with the members of ICSM ePlan Working Group from Australia, New Zealand and Singapore in face to face meetings to ensure that other jurisdictions can also benefit from the strategic actions and initiatives experienced in Victoria.

As the initiatives suggested by the framework become more established, future investigations can focus on examining the impacts of strategic actions on the uptake of ePlan. In parallel with the implementation of the proposed framework, a study on the human aspects of the non-adoption may also be required.

Acknowledgments: The authors would like to thank the ICSM ePlan Working Group, LUV staff, and the Centre for Spatial Data Infrastructures and Land Administration www.csdila.unimelb.edu.au at the University of Melbourne for sharing their thoughts and contributing to this paper. This paper is based on the view of the authors and may not represent the view of LUV.

Author Contributions: Hamed Olfat, Davood Shojaei and Mark Briffa ran the interviews with surveyors and analyzed the outcomes. They also developed the framework of strategic actions and initiatives and wrote the manuscript. Susannah Maley provided the content for SPEAR portal. Abbas Rajabifard contributed to the development of the strategic actions and initiatives and provided feedback throughout the project.

Conflicts of Interest: The authors declare no conflict of interest.

\section{References}

1. Rajabifard, A. Cadastre 2014 in Relation to Spatial Data Infrastructure (SDI). In CADASTRE 2014 and Beyond; Steudler, D., Ed.; FIG Publication No. 61; FIG: Copenhagen Denmark, 2014; pp. 49-53.

2. Falzon, K.; Williamson, I. Digital lodgment of cadastral survey data in Australia-User needs. Trans. Tasman. Surv. J. 2001, 4, 8-17.

3. ICSM Cadastre 2034 Strategy, Powering Land and Property. 2015. Available online: http:/ /www.icsm.gov. au/cadastral/Cadastre2034.pdf (accessed on 2 May 2017).

4. Aien, A.; Kalantari, M.; Rajabifard, A.; Williamson, I.; Shojaei, D. Developing and testing a 3D cadastral data model: A case study in Australia. In Proceedings of the XXII ISPRS Congress, ISPRS Annals of the Photogrammetry, Melbourne, Australia, 25 August-1 September 2012.

5. Cumerford, N. The ICSM ePlan protocol, its development, evolution and implementation. In Proceedings of the XXIV FIG Congress, Sydney, Australia, 11-16 April 2010.

6. Haanen, A.; Bevin, T.; Sutherland, N. e-Cadastre-Automation of the New Zealand Survey System. In Proceedings of the FIG XXII International Congress, Washington, DC, USA, 19-26 April 2002.

7. Bevin, T. e-Survey and Title in New Zealand-Landonline. In Proceedings of the Joint AURISA and Institution of Surveyors Conference, Adelaide, Australia, 25-30 November 2002.

8. LINZ Cadastral Survey (Compulsory Lodgment of Digital Cadastral Survey Datasets) Order 2007. Available online: http:/ / www.legislation.govt.nz/regulation/public/2007/0215/latest/DLM444505.html?search= ts_regulation\%40deemedreg_cadastral_resel_25_a\&p=1 (accessed on 2 May 2017).

9. Soon, K.H. A conceptual framework of representing semantics for 3D cadastre in Singapore. In Proceedings of the 3rd International FIG Workshop on 3D Cadastres: Developments and Practices, Shenzhen, China, 25-26 October 2012.

10. Thompson, R.J.; Van Oosterom, P.; Soon, K.H. LandXML Encoding of Mixed 2D and 3D Survey Plans with Multi-Level Topology. ISPRS Int. J. Geo-Inf. 2017, 6, 171. [CrossRef]

11. Singapore LandXML Mapping and Structure. Available online: https://www.sla.gov.sg/Portals/0/ SurveyServices/SG\%20LandXML/SGLX_Specification_1.3.pdf?ver=2017-05-05-104305-997 (accessed on 11 January 2018).

12. Soon, K.H.; Tan, D.; Khoo, V. Initial Design to Develop a Cadastral System that Supports Digital Cadastre, 3D and Provenance for Singapore. In Proceedings of the 5th International FIG Workshop on 3D Cadastre, Athens, Greece, 18-20 October 2016. 
13. ISO 19152:2012, Geographic Information-Land Administration Domain Model (LADM); ISO: Geneva, Switzerland, 2012.

14. Kalantari, M.; Dinsmore, K.; Urban-Karrb, J.; Rajabifard, A. A roadmap to adopt the Land Administration Domain Model in cadastral information systems. Land Use Policy 2015, 49, 552-564. [CrossRef]

15. De Almeida, J.; Ellul, C.; Rodrigues-De-Carvalho, M. Towards a Real Estate Registry 3D Model in Portugal: Some Illustrative Case Studies, ISPRS Annals of the Photogrammetry, Remote Sensing and Spatial Information Sciences. In Proceedings of the ISPRS 8th 3DGeoInfo Conference \& WG II/2 Workshop, Istanbul, Turkey, 27-29 November 2013; Volume II-2/W1.

16. Elia, E.; Zevenbergen, J.; Lemmen, C.; Van Oosterom, P. The land administration domain model (LADM) as the reference model for the Cyprus land information system (CLIS). Surv. Rev. 2013, 45, 100-110.

17. Pouliot, J.; Vasseur, M.; Boubehrezh, A. How the ISO 19152 Land Administration Domain Model performs in the comparison of cadastral systems: A case study of condominium/co-ownership in Quebec (Canada) and Alsace Moselle (France). Comput. Environ. Urban Syst. J. 2013, 40, 68-78. [CrossRef]

18. Santos, J.C.D.; Farias, E.S.D.; Carneiro, A.F.T. Analysis of the parcel as a land unity of the Brazilian urban cadastre. Bol. Ciênc. Geod. 2013, 19, 574-587. [CrossRef]

19. Tjia, D.; Coetzee, S. Application of the Land Administration Domain Model to the city of Johannesburg land information system. S. Afr. J. Geomat. 2013, 2, 260-279.

20. Vučić, N.; Markovinović, D.; Mičević, B. LADM in the Republic of Croatia-Making and testing country profile. In Proceedings of the 5th Land Administration Domain Model Workshop, Kuala Lumpur, Malaysia, 24-25 September 2013.

21. Permanent Committee on Cadastre in the European Union. Overview on the Cadastral Systems of the E.U. Member States Part III; Lantmäteriet: Gävle, Sweden, 2009.

22. Permanent Committee on Cadastre in the European Union. Overview on the Cadastral Systems of the E.U. Member States; Sogei: Rome, Italy, 2008.

23. Victorian Government Torrens Titles 2017. Available online: http://www.dtpli.vic.gov.au/property-andland-titles/land-titles/torrens-titles (accessed on 3 May 2017).

24. Victorian Government Planning and Environment Act 1987. 2017. Available online: http: / / www.legislation.vic.gov.au/Domino/Web_Notes/LDMS/LTObject_Store/LTObjSt7.nsf/ 07c00f1b6c5c4afbca25776700219570/4d5c8a5d22cc9998ca257a3000028d7a/\$FILE/87-45aa102\% 20authorised.pdf (accessed on 3 May 2017).

25. Victorian Government Subdivision Act 1988. 2017. Available online: http://www.legislation.vic.gov.au/ Domino/Web_Notes/LDMS/LTObject_Store/LTObjSt5.nsf/DDE300B846EED9C7CA257616000A3571/ 4DE5957BD473E397CA25781B00180F22/\$FILE/88-53aa065\%20authorised.pdf (accessed on 3 May 2017).

26. Olfat, H.; Shojaei, D.; Briffa, M.; Rajabifard, A. The Current Status and Ongoing Investigations of 2D and 3D Digital Cadastre (ePlan) in Victoria, Australia. In Proceedings of the Academic Research Stream at the Annual Conference Locate, Research@Locate 2017 co-Located with Digital Earth \& Locate 2017, Sydney, Australia, 3-6 April 2017.

27. Myers, M.D. Qualitative Research in Information Systems. MIS Q. 1997, 21, 241-242. [CrossRef]

28. Maxwell, J.A. Qualitative Research Design: An Interactive Approach; Sage Publications: Thousand Oaks, CA, USA, 1996.

29. Mack, N.; Woodsong, C.; MacQueen, K.M.; Guest, G.; Namey, E. Qualitative Research Methods: A Data Collector's Field Guide; Family Health International: Durham, NC, USA, 2005.

30. Hartley, J. Case Study Research. In Essential Guide to Qualitative Methods in Organizational Research; Cassell, C., Symon, G., Eds.; SAGE Publications Ltd.: London, UK, 2004.

31. Opdenakker, R. Advantages and disadvantages of four interview techniques in qualitative research. Forum Qual. Soc. Res. 2006, 7. [CrossRef]

32. SKM Business Case for a Spatially Accurate Map Base 2011. Available online: http://studylib.net/doc/ 7609543/business-case-for-a-spatially-accurate-map-base-2011--doc (accessed on 2 May 2017).

33. Shojaei, D.; Olfat, H.; Foundez, S.; Kalantari, M.; Rajabifard, A.; Briffa, M. Geometrical data validation in 3D digital cadastre-A case study for Victoria, Australia. Land Use Policy 2017, 68, 638-648. [CrossRef]

34. Shojaei, D.; Olfat, H.; Rajabifard, A.; Darvill, A.; Briffa, M. Assessment of the Australian digital cadastre protocol (ePlan) in terms of supporting 3D building subdivisions. Land Use Policy 2016, 56, 112-124. [CrossRef] 
35. Shojaei, D.; Olfat, H.; Briffa, M.; Rajabifard, A. 3D Cadastre Journey in Victoria, Australia. In Proceedings of the ISPRS Annals of the Photogrammetry, Remote Sensing and Spatial Information Sciences, Melbourne, Australia, 26-27 October 2017; Volume IV-4/W5, pp. 117-123.

36. Ho, S. Towards 3D-Enabled Urban Land Administration: Invisible Constraints and Strategic Choices. Ph.D. Thesis, the University of Melbourne, Parkville, Australia, 2014.

37. Kalantari, M.; Rajabifard, A. A Roadmap to Accomplish 3D Cadastres. In Proceedings of the 4th International Workshop on 3D Cadastres, Dubai, UAE, 9-11 November 2014.

38. Ho, S.; Rajabifard, A. Towards 3D-enabled urban land administration: Strategic lessons from the bim initiative in Singapore. Land Use Policy 2016, 57, 1-10. [CrossRef]

(C) 2018 by the authors. Licensee MDPI, Basel, Switzerland. This article is an open access article distributed under the terms and conditions of the Creative Commons Attribution (CC BY) license (http://creativecommons.org/licenses/by/4.0/). 